\title{
Force-Field Development from Electronic Structure Calculations with Periodic Boundary Conditions: Applications to Gaseous Adsorption and Transport in Metal-Organic Frameworks
}

Li-Chiang Lin, ${ }^{*, \dagger, \nabla}$ Kyuho Lee, ${ }^{*, \dagger, \dagger, \nabla}$ Laura Gagliardi, ${ }^{\S}$ Jeffrey B. Neaton, ${ }^{\ddagger} \|$ and Berend Smit ${ }^{\dagger, \perp, \#}$

${ }^{\dagger}$ Department of Chemical and Biomolecular Engineering, University of California, Berkeley, Berkeley, California, 94720, United States

${ }^{\ddagger}$ Molecular Foundry, Lawrence Berkeley National Laboratory, Berkeley, California, 94720, United States

${ }^{\S}$ Department of Chemistry, Supercomputing Institute, and Chemical Theory Center, University of Minnesota, Minneapolis, Minnesota, 55455, United States

"Department of Physics, University of California, Berkeley, Berkeley, California, 94720, United States

${ }^{\perp}$ Department of Chemistry, University of California, Berkeley, Berkeley, California, 94720, United States

${ }^{\#}$ Materials Sciences Division, Lawrence Berkeley National Laboratory, Berkeley, California, 94720, United States

Supporting Information

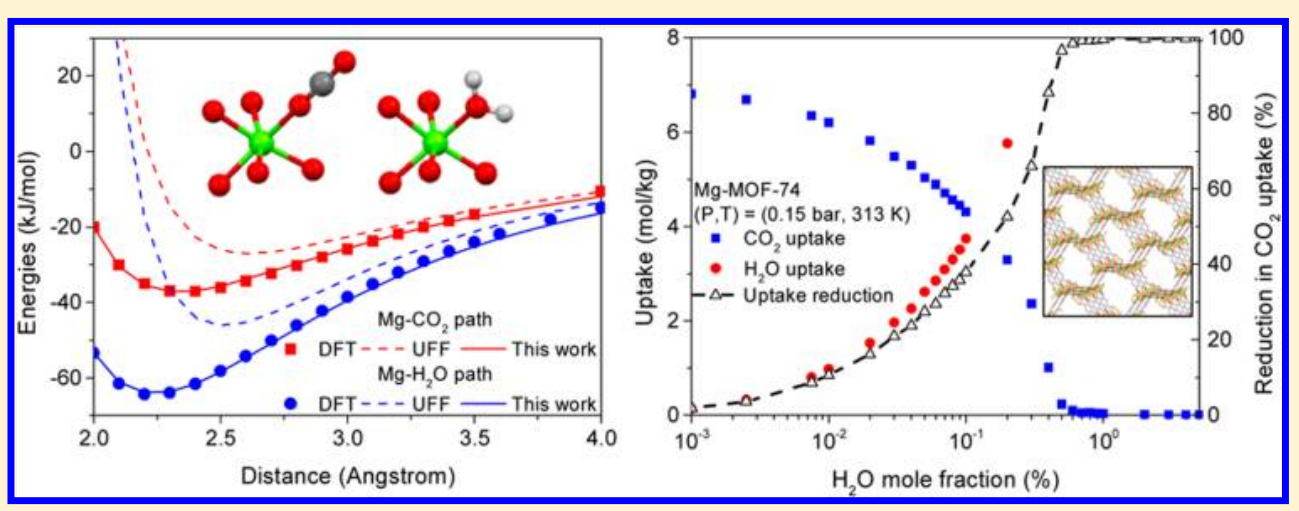

ABSTRACT: We present a systematic and efficient methodology to derive accurate (nonpolarizable) force fields from periodic density functional theory (DFT) calculations for use in classical molecular simulations. The methodology requires reduced computation cost compared with other conventional ways. Moreover, the whole process is performed self-consistently in a fully periodic system. The force fields derived by using this methodology nicely predict the $\mathrm{CO}_{2}$ and $\mathrm{H}_{2} \mathrm{O}$ adsorption isotherms inside Mg-MOF-74, and is transferable to $\mathrm{Zn}-\mathrm{MOF}-74$; by replacing the $\mathrm{Mg}_{-} \mathrm{CO}_{2}$ interactions with the corresponding $\mathrm{Zn}-\mathrm{CO}_{2}$ interactions, we obtain an accurate prediction of the corresponding isotherm. We have applied this methodology to address the effect of water on the separation of flue gases in these materials. In general, the mixture isotherms of $\mathrm{CO}_{2}$ and $\mathrm{H}_{2} \mathrm{O}$ calculated with these derived force fields show a significant reduction in $\mathrm{CO}_{2}$ uptake with the existence of trace amounts of water vapor. The effect of water, however, is found to be quantitatively different between $\mathrm{Mg}$ - and $\mathrm{Zn}-\mathrm{MOF}-74$.

\section{INTRODUCTION}

Metal-organic frameworks (MOFs) are a relatively new class of porous materials first synthesized about a decade ago and have become of great interest to the chemistry and materials community. ${ }^{1}$ Due to their large internal surface area and enhanced adsorption capacity, MOFs are highly promising for more energetically efficient carbon capture and sequestration (CCS) applications. ${ }^{2-4}$ CCS is one of the few viable ways to reduce the emission of $\mathrm{CO}_{2}$ into the atmosphere and to mitigate green house effects associated with the continuous use of fossil fuels. ${ }^{5}$ One of the attractive features of MOFs is that they are highly tunable materials, and one can possibly synthesize an ideal framework by using exactly the right combination of metal cluster and organic linker for optimal performance. Since the number of distinct MOFs that one can envision is essentially infinite, finding such an optimal combination is a great challenge, as it is practically impossible to synthesize and characterize all possible materials experimentally. In this regard, it would be helpful to evaluate the performance of these materials by molecular simulation techniques prior to experimental synthesis. A prerequisite to making reliable predictions of relevant thermodynamic and transport properties of gas molecules adsorbed in these

Received: February 6, 2014

Published: February 25, 2014 


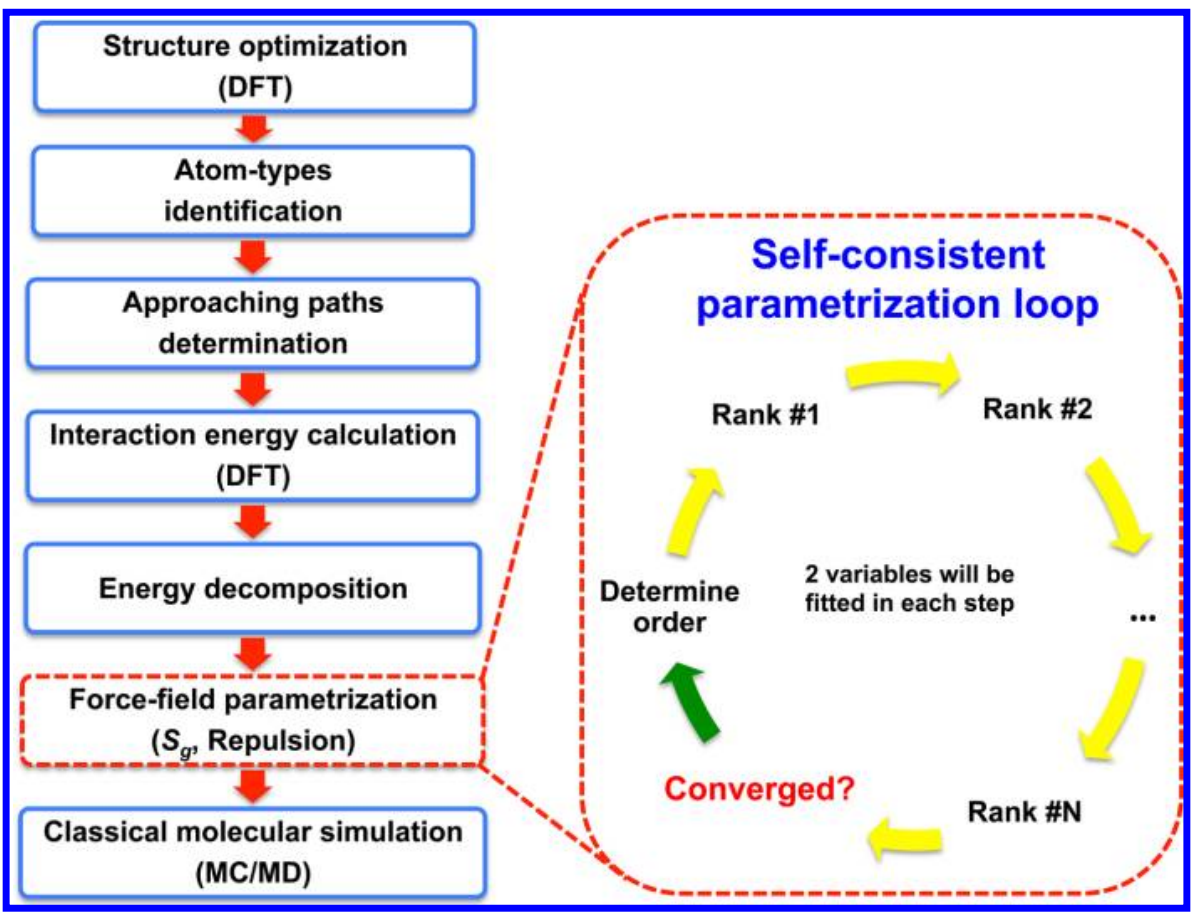

Figure 1. Flow diagram of the proposed method to derive force field from periodic DFT calculations.

materials is that we can accurately describe the interactions of these gas molecules with the atoms of the materials. Namely, there is a necessity to have accurate force fields for use in molecular simulations.

Reasonable estimates of the thermodynamic and transport properties of gases in MOFs might be obtained using one of the common force fields (e.g., universal force field (UFF) ${ }^{6}$ or Dreiding $\left.{ }^{7}\right)$. However, the chemical diversity of MOFs includes many new types of functionalities for which these force fields fail to give a reasonable description. Of particular interest is the subset of MOFs with so-called open-metal sites. ${ }^{8}$ During the crystallization of such MOFs from a solution, solvent molecules can directly coordinate to the metal sites. Once the MOF crystals are formed, the material can be activated by removing these solvent molecules, leaving under-coordinated open-metal sites. The open-metal sites are responsible for some unique properties. For instance, M-MOF-74, also known as M-CPO27, frameworks (e.g., $\mathrm{M}=\mathrm{Mg}, \mathrm{Zn}$, etc.) have been demonstrated to have extraordinarily high $\mathrm{CO}_{2}$ adsorption capacity and selectivity toward $\mathrm{CO}_{2}$ over $\mathrm{N}_{2}$ under flue gas conditions, making them to be promising candidates for CCS applications. ${ }^{9-11}$ From a computational point of view, it has been shown that these common force fields provide only a limited description of the interaction strength between guest molecules and these open-metal site structures and therefore yield a poor estimation of their adsorption properties. ${ }^{12}$

A few studies have been reported in the literature to improve the accuracy of force fields for these systems using ab initio calculations. Chen et al. ${ }^{13}$ utilized a genetic algorithm to parametrize dozens of force-field parameters for $\mathrm{CO}_{2}$ in $\mathrm{Mg}$ MOF-74. This method relied on many (thousand) single-point quantum-chemical calculations. Fang et al. proposed a methodology to obtain force fields from dispersion-corrected density functional theory (DFT) calculations, and the method accurately predicts the $\mathrm{CO}_{2}$ adsorption isotherms inside zeolites. ${ }^{14}$ In their approach, two global scaling factors were added to the classical 12-6 Lennard-Jones potential, and these parameters were optimized using about one thousand singlepoint DFT calculations. Zang et al. used this method to study $\mathrm{H}_{2} \mathrm{O}$ inside $\mathrm{CuBTC}$, which also possesses open-metal sites. ${ }^{15}$ Zang et al. observed, however, that for this material appropriate scaling factors could not be determined. Instead, an empirical term as a function of the copper-water distance was added to correct the total energies computed by common force fields.

In a previous work, some of us proposed a methodology to develop force fields generalizable to a broad class of MOFs including open-metal site structures, and the methodology required only a small number of single-point $a b$ initio calculations. ${ }^{16}$ This method utilized Møller-Plesset secondorder perturbation theory (MP2) cluster calculations for each configuration along approaching paths that were designed to probe specific pairwise interaction energies. The fitting procedure was further simplified by decomposing the MP2calculated energies into electrostatic, repulsive, and attractive interactions using the nonempirical model potential (NEMO) decomposition technique. ${ }^{17,18}$ As a result, a simple fitting algorithm (i.e., grid search method) was required as only up to two variables have to be optimized at the same time. For instance, a $\mathrm{CO}_{2}$ force field inside Mg-MOF-74 was obtained from about one hundred designed configurations, and the predicted adsorption isotherm was found to be in excellent agreement with the experimental data.

Compared to DFT calculations with periodic boundary conditions, MP2 calculations require significantly more computational resources and are therefore limited to smaller clusters. In addition, the NEMO technique was developed for molecular systems. Consequently, the previously developed force-field parameters were based upon cluster calculations. ${ }^{16}$ By increasing the size of these clusters, one can systematically improve the description of extended (periodic) systems. However, conventional MP2 calculations scale as $\mathrm{N}^{6}$ and are unaffordable for large clusters (i.e., $>100$ atoms). This poses practical limitations on the approach. As a result, this force field could not correctly predict the binding geometry of the $\mathrm{CO}_{2}$ 
molecule in Mg-MOF-74 as measured, for example, with neutron diffraction. ${ }^{19}$ Another important conclusion of our previous work is that MP2 and van der Waals dispersioncorrected DFT (i.e., vdW-DF) give nearly identical results at the cluster level for the Mg-MOF-74 system. ${ }^{16}$ The agreement between these two approaches is not surprising, because $\mathrm{Mg}$ (II) is a closed-shell system and the interaction between $\mathrm{CO}_{2}$ and $\mathrm{Mg}$ is dominated by dispersion-effects. It should be noted that, if open-shell transition metals (e.g., $\mathrm{Fe}(\mathrm{II})$ ) were considered, one would have to be more careful because stronger electron correlation effects may occur and have to choose an appropriate functional or use multireference wave function based methods. Accordingly, all of above suggests that we should extend our methodology to periodic systems to remove all these constraints imposed by the use of cluster calculations and develop a force field that not only correctly predicts the adsorption isotherms but also yields accurate binding geometries.

In this work, we propose a new approach for deriving force fields from periodic DFT calculations. This enables all steps of the force-field generation to be conducted within a periodic framework: from the structural optimization of the MOFs, to the force-field parametrization, to classical molecular simulations (i.e., Monte Carlo and molecular dynamics). With this newly developed methodology, the computational time required to obtain a DFT-derived force field is highly reduced, about the same order of magnitude of the cost required for periodic structure relaxation. We have used this newly developed methodology to obtain an improved force field for $\mathrm{CO}_{2}$ in Mg-MOF-74, and our new force field can accurately predict the $\mathrm{CO}_{2}$ binding geometry, adsorption isotherm, and dynamic property inside the material. We further demonstrate that our methodology is applicable for generating force fields for water molecules within MOFs. Water is one of the major components in the flue gases emitted from coal-fired power plants, and the presence of water can potentially degrade the $\mathrm{CO}_{2}$ adsorption performance enormously. ${ }^{20,21}$ In this context, it is crucial to consider the effect of water on $\mathrm{CO}_{2}$ uptake while evaluating the performance of a given MOF. Using our new $\mathrm{ab}$ initio force fields, we have studied the performance of $\mathrm{CO}_{2}$ adsorbed inside M-MOF-74 $(\mathrm{M}=\mathrm{Mg}$ and $\mathrm{Zn})$ in the presence of water vapor.

\section{METHODOLOGY}

Overview. The methodology consists of the following steps (see Figure 1 for the flow diagram)

- Structure optimization: Periodic DFT structural optimization is utilized to obtain reliable geometries, which will be used throughout the entire process.

- Atom-types identification: For a given structure, we identify different atom-types on the framework for which we need to determine the pairwise potentials.

- Approaching paths determination: For each of the identified atom-types, we determine an approaching orientation (path) toward the atom-type and a set of configurations of the guest molecule along the orientation.

- Interaction energy calculation: For each configuration, the interaction energy between the guest molecule and the MOF is computed using DFT with an appropriate dispersion-corrected exchange-correlation functional.
- Energy decomposition: The total interaction energies are decomposed into three major contributions: long-range Coulomb interactions, short-range exchange repulsion, and dispersion interactions.

- Force-field parametrization: The force field is then parametrized with the guest-framework short-range repulsive parameters being optimized. In this step, we have introduced a self-consistently fitting loop to ensure that the force field becomes independent of the details of the energy decomposition.

- Classical molecular simulation: The obtained force field is used as the basis in classical molecular simulations for adsorptive and diffusive property predictions. These molecular simulations also require a force field for the guest-guest interactions. For these, we directly employ one of the existing models that can reproduce the experimental guest molecular properties, such as vaporliquid equilibrium (VLE).

Each step is discussed in detail in the following sections. We use $\mathrm{CO}_{2}$ adsorbed in Mg-MOF-74 as case study.

Structure Optimization (DFT). For some materials, the experimental crystal structures rely on powder samples for which the accuracy of obtained atomic positions can be very limited. For instance, Dzubak et al. have shown that the experimentally obtained structure of Mg-MOF-74 has distorted benzene rings, and the computed $\mathrm{CO}_{2}$ adsorption properties inside the material are very sensitive to the details of the adopted structure. ${ }^{16}$ Accordingly, DFT, which can capture many details of the structure with good accuracy, is utilized to ensure the force fields are faithful to result from accurate crystal structures. In this work, we use the same optimized geometries as in our previous work. ${ }^{16}$

Atom-Types Identification and Approaching Paths Determination. M-MOF-74 is composed of four different elements: Metal (M), O, C, and $\mathrm{H}$. If one further considers the local environment of each of these elements, 9 different atomtypes can be identified, denoted as $\mathrm{M}, \mathrm{O}_{a}, \mathrm{O}_{\mathrm{b}}, \mathrm{O}_{\mathcal{c}} \mathrm{C}_{a}, \mathrm{C}_{\mathrm{b}}, \mathrm{C}_{\mathcal{c}}$ $\mathrm{C}_{\mathrm{d}}$, and $\mathrm{H}$ (see Supporting Information (SI) Figure S1). Note that the hydrogen in this case is not considered explicitly, and thus, there is no corresponding path to be considered.

In our approach, the short-range repulsive forces between each nonbonding pair of framework atom-types and guest molecule are specifically probed. For each framework atomtype, the method determines an approaching orientation (path) of a given guest molecule toward the target atom-type. This approaching orientation is determined by minimizing the repulsive energy between the guest molecule and the framework atoms with other atom-types (i.e., different from the target atom-type). This procedure ensures that the pairwise repulsive interaction between the guest molecule, particularly the closest atom, and the target atom dominates the total repulsive energy. For instance, the repulsive interaction between the $\mathrm{O}\left(\mathrm{CO}_{2}\right)$ and the $\mathrm{Mg}$ atom-type of the framework is designed to be the dominant component of the total repulsion along the corresponding $\mathrm{Mg}-\mathrm{CO}_{2}$ path. The combination (i.e., $\mathrm{O}\left(\mathrm{CO}_{2}\right)-\mathrm{Mg}$ ) is the so-called "approached pair" along a given path. UFF force fields are used to determine these paths. For each path, $\sim 15$ configurations at different distances (i.e., with the intervals of 0.1 or $0.2 \AA$ ) from the approached atom-type are generated.

Ideally, one would like to be able to generate specific paths for all atoms of the guest molecule (e.g., $\mathrm{C}$ and $\mathrm{O}$ of $\mathrm{CO}_{2}$ ) with 
respect to all atom-types of the MOF. However, for some of the atom combinations, we cannot generate a path for which the contribution of the approached pair cannot dominate the total repulsion. We denote these atom combinations as "unapproachable pairs". In this case, the unapproachable pairs are those pairs that involve the carbon atom of $\mathrm{CO}_{2}$.

Interaction Energy Calculation (DFT). The interaction energies of an individual guest molecule inside the MOF (i.e., $\left.E_{\text {int }}\right)$ are defined as

$$
E_{\text {int }}=E_{(\mathrm{MOF}+\text { guest })}-\left(E_{(\mathrm{MOF})}+E_{(\text {guest })}\right)
$$

All the calculations are conducted in the conventional hexagonal unit cell composed of 162 atoms. We use DFT with vdW-DF2 exchange-correlation functional ${ }^{22}$ for all total energy calculations as implemented in the Quantum Espresso $\operatorname{code}^{23}$ with a planewave basis. We use the Troullier-Martins type norm-conserving pseudopotentials, and kinetic energy and charge density planewave cutoff are set to be $120 \mathrm{Ry}$ and 480 Ry, respectively. The integration over the irreducible Brillouin zone is carried out over $2 \times 2 \times 4$ Monkhorst-Pack grids.

Force Field. We use two different types of potential forms for the short-range repulsion: the Lennard-Jones type $1 / r^{12}$ and the Buckingham type $e^{-r}$ potential as shown in eqs 2 and 3 , respectively.

$$
\begin{aligned}
& E_{i j}=\frac{q_{i} q_{j}}{4 \pi \varepsilon_{o} r_{i j}}+4 \varepsilon_{i j}\left[\left(\frac{\sigma_{i j}}{r_{i j}}\right)^{12}-\left(\frac{\sigma_{i j}}{r_{i j}}\right)^{6}\right] \\
& E_{i j}=\left\{\begin{array}{c}
\infty \quad \text { if } \quad r_{i j}<R_{\text {min }} \\
\frac{q_{i} q_{j}}{4 \pi \varepsilon_{o} r_{i j}}+A_{i j} e^{-B_{i j} r_{i j}}-S_{g} \frac{C_{i j}}{r_{i j}^{6}} i r_{i j} \geq R_{\text {min }}
\end{array}\right\}
\end{aligned}
$$

where $q_{i}$ and $q_{j}$ are the atomic charges of atoms $i$ and $j, r_{i j}$ is the distance between atoms $i$ and $j, \varepsilon_{o}$ is the permittivity of free space, $R_{\min }$ is used to prevent the conventional Buckingham potential from going to negative infinity at short distance, $\varepsilon_{i j}$ and $\sigma_{i j}$ are the Lennard-Jones energy and distance parameters, respectively, $\left(A_{i j}, B_{i j}\right)$ and $C_{i j}$ are the conventional Buckingham parameters for repulsive and attractive contribution, respectively. In this work, we introduce a global scaling factor, $S_{g}$, for the dispersion energies. The rationale for this factor will be discussed in one of the next sections. It should be noted that nonelectrostatic pair-wise potential will be truncated and shifted to zero at the cutoff radius of $12.8 \AA$ while computing interaction energies in periodic system.

It is well known that the repulsive interactions can be described better with the Buckingham potential (i.e., $e^{-r}$ ) compared to the Lennard-Jones form (i.e., $1 / r^{12}$ ) (e.g., see Dzubak et al. ${ }^{16}$ ) Therefore, we use the Buckingham potential for all approachable pairs. In the next section, we discuss how we obtain the parameters of the Buckingham potential. For the pairs that are unapproachable (e.g., include $\mathrm{C}\left(\mathrm{CO}_{2}\right)$ ) or not considered explicitly (e.g., framework atom-type $\mathrm{H}$ ), we keep the Lennard-Jones potential with parameters given from one of the common force fields.

Energy Decomposition. The DFT-computed energies for these configurations are decomposed into long-range Coulomb interactions, short-range exchange repulsion, and dispersion interactions. The long-range Coulomb contribution is computed through the Ewald summation technique. ${ }^{24}$ The partial atomic charges of the framework atoms are determined using the REPEAT scheme, ${ }^{25}$ with the self-consistent DFT electrostatic potentials obtained from the DFT structural relaxation. The REPEAT scheme finds a set of atomic charges that best represents the DFT electrostatic potential surface. The charges for the guest molecules are directly taken from the guest molecule models (e.g., $\mathrm{TraPPE}^{26}$ or $\mathrm{EPM}^{27}$ models for $\mathrm{CO}_{2}$ molecules).

The dispersion term is computed with $C_{6}$ dispersion coefficients that are initially taken from one of the commonly used force fields. We assume that these coefficients give reasonable relative energies but their absolute values can be off. To correct this, we introduce a global scaling factor, $S_{g}$. We can then obtain the decomposed short-range repulsion by subtracting from the total energies the sum of the dispersive and electrostatic energies. Note that without the scaling factor, this decomposition can result in negative repulsive energies (see SI Figure S2). Namely, a global scaling factor is determined to ensure the decomposed repulsion energies are all positive-definite along each path.

Force-Field Parametrization. The methodology utilizes the short-range nature of the repulsive energies, and probes each pairwise interaction separately (i.e., different paths for different approached pair). Given that the specific repulsion between the approached pair along a given path is designed to dominate the total repulsion, only the corresponding pairwise repulsive parameters (i.e., 2 parameters: $A_{i j}$ and $B_{i j}$ ) are optimized by grid search method to reproduce the total decomposed repulsion energies along the path. Further, to determine the order in which we fit these parameters of different approached pairs, we compute the ratio of the computed repulsive energy between the approached pair to the total repulsive energy for each path. The fitting of the pairwise repulsive parameters is then performed for each path sequentially in order from the highest to lowest ratio. This procedure is repeated iteratively until all the parameters are converged (i.e., no change in parameters after a loop). With the fitted parameters of a given global scaling factor, the fitting can be further fine-tuned to obtain the optimal scaling factor by minimizing the error, e.g., difference between the energies computed by the force field and by DFT along all the paths.

Classical Molecular Simulation. Monte Carlo (MC) and molecular dynamics (MD) simulations are used for predicting thermodynamic and transport properties of the adsorbed gases. In these simulations, total interaction energies include the electrostatics, short-range repulsion, and dispersion energies using the force-field parameters obtained from the previous section. Nonelectrostatic potential is truncated and shifted to zero at the cutoff radius of $12.8 \AA$. The simulation box is composed of multiple unit cells to ensure that each distance between two successive planes of $(1,0,0),(0,1,0)$, and $(0,0,1)$ is at least twice the cutoff radius. Detailed information of the force-field parameters can be found in SI section 3 .

For MC simulations in the grand-canonical ensemble (i.e., GCMC simulations), several million configurations are sampled. These configurations are generated by random translation, rotation, insertion, and deletion to obtain statistically averaged loadings as a function of the fugacity with high accuracy. The MD simulations are carried out in the canonical ensemble and use the Nosé-Hoover thermostat with a time step of $0.5 \mathrm{fs}$ for a total simulation time of at least $10 \mathrm{~ns}$. 

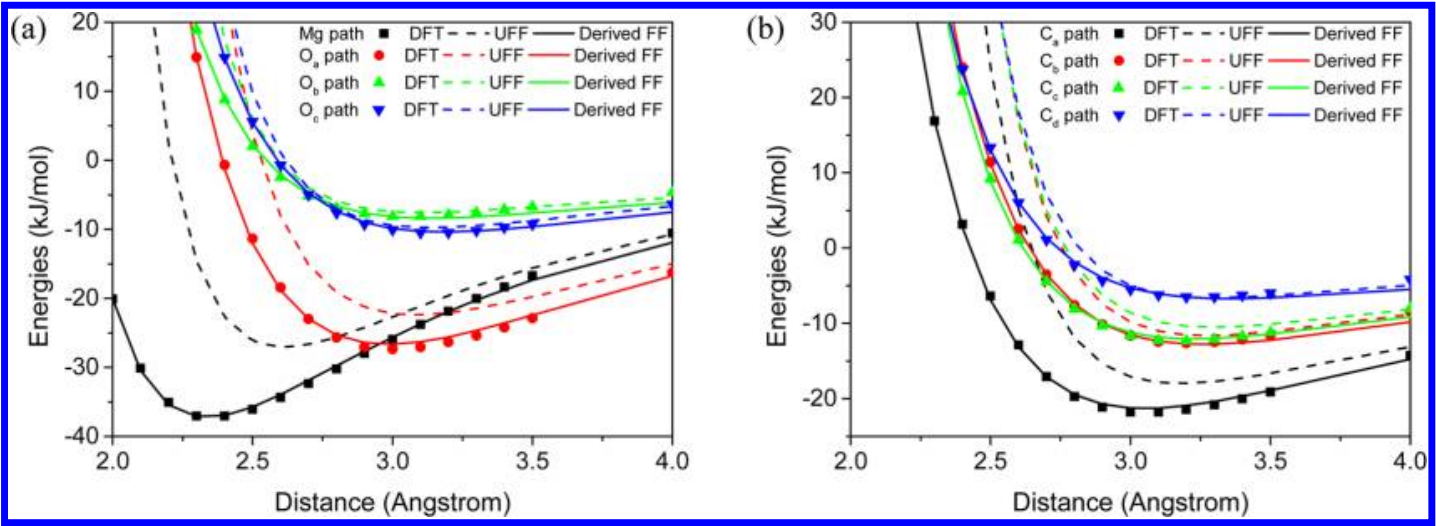

Figure 2. Comparisons of $\mathrm{CO}_{2}$-framework interaction energies as a function of distance inside Mg-MOF-74 obtained from the DFT calculations (closed symbols) with the ones computed from the DFT-derived force field (model 1, solid line) and the UFF (dashed line) along (a) Mg and O and (b) C paths.

\section{RESULT AND DISCUSSION}

$\mathrm{CO}_{2}$ Adsorption and Dynamics in Mg-MOF-74. To validate our approach, we first derive a force field for $\mathrm{CO}_{2}$ adsorbed in Mg-MOF-74. The EPM2 model is adopted for $\mathrm{CO}_{2}$ molecules while UFF force fields are used for framework atoms as a starting point. The resulting DFT-derived force field is denoted as model 1 .

Thermodynamic Properties. Figure 2 shows that the model 1 nicely reproduces the DFT energies along all the paths. Comparison with the energies computed with the original UFF force field shows that UFF overestimates the short-range repulsive energies and, therefore, underestimates the interaction energies. To further evaluate our new force field, a total of 1200 random configurations are generated in the accessible pore volume. These points are not included in the fitting procedure. The comparison between the DFT computed energies and the force-field energies for these random configurations is given in Figure 3. The green-dashed line represents a perfect agreement between the DFT and force-field energies, and the energies computed by DFT-derived force field nicely lie on top of the line. In contrast, the energies computed by UFF tend to underestimate the interaction energies. To further quantify the

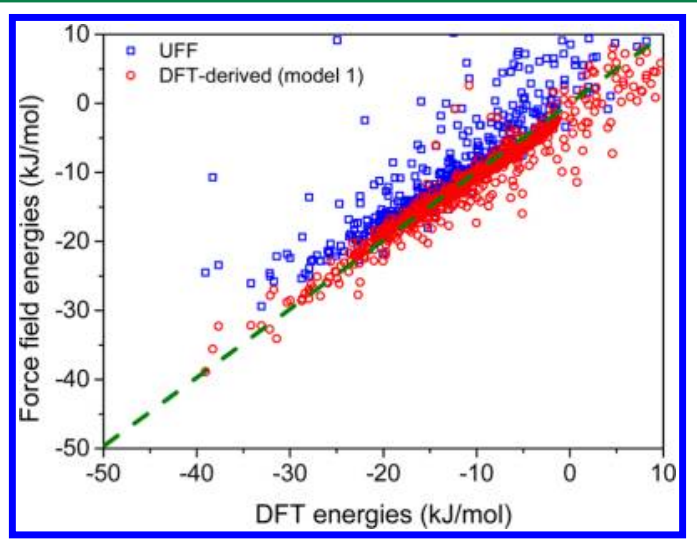

Figure 3. Comparison of the $\mathrm{CO}_{2}-$ framework interaction energies obtained from the DFT calculations with the ones computed from the UFF (open blue squares) and the DFT-derived force field (model 1, open red circles) for a set of $1200 \mathrm{CO}_{2}$ random configurations inside the accessible pore volume of Mg-MOF-74. The green-dashed line indicates perfect agreement between the energies computed by DFT and by force fields. improvement, we compute the Boltzmann-weighted mean deviation (BMD) that is defined as eq 4 :

$$
\mathrm{BMD}=\frac{\sum_{i=1}^{N_{s}}\left(E_{\mathrm{FF}, i}-E_{\mathrm{DFT}, i}\right) \exp \left(-\frac{E_{\mathrm{DF}, i}}{k_{\mathrm{B}} T}\right)}{\sum_{i=1}^{N_{s}} \exp \left(-\frac{E_{\mathrm{DFF}, i}}{k_{\mathrm{B}} T}\right)}
$$

where $N_{s}$ is the total number of sampled configurations while $E_{\mathrm{FF}, i}$ and $E_{\mathrm{DFT}, i}$ represent the interaction energies of configuration $i$ computed by force fields and DFT, respectively. The Boltzmann-weighted mean deviations at $T=300 \mathrm{~K}$ for the UFF and DFT-derived FF (model 1) are 15.27 and $2.02 \mathrm{~kJ} /$ mol, respectively.

In Figure 4, we compare the adsorption isotherms of model 1 with the experimental isotherms for $\mathrm{CO}_{2}$ inside Mg-MOF-74. Since it is well known that not all the $\mathrm{Mg}$ sites are accessible, we rescale the experimental isotherm by assuming $80 \%$ availability of the materials. ${ }^{28}$ The determination of the scaling factor is based on the inflection point of the experimentally measured heat of adsorption as a function of adsorbed $\mathrm{CO}_{2}$ loading.

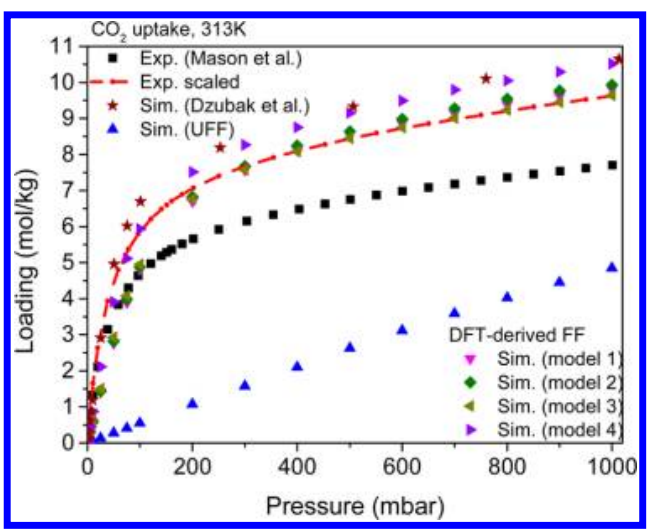

Figure 4. Comparison between the experimental and simulated isotherms of $\mathrm{CO}_{2}$ inside Mg-MOF-74 at $313 \mathrm{~K}$. The experimental data of Mason et al..$^{28}$ and the corresponding scaled isotherm (assume $80 \%$ of material is accessible) are shown by the closed black squares and red closed circles with dashed line, respectively. The blue closed uppertriangles show the predicted isotherm using the UFF force field while the brown closed stars show the predicted isotherm by using the MP2derived force field of Dzubak et al. ${ }^{16}$ The other symbols correspond to the isotherms of four different DFT-derived force fields (models 1 to $4)$. 
Detailed discussion can be found in ref 16 . With our new DFTderived force field, the predicted isotherm is in excellent agreement with the scaled experiment isotherm.

At this point, it is instructive to compare our new force field with the one we previously derived from MP2-cluster calculations. The predicted adsorption isotherms by this MP2 force field are in good agreement with the experimental data (see Figure 4). ${ }^{16}$ However, if we compare the MP2 force-field predictions for a set of random configurations with the DFT energies (see SI Figure S3), we observe that the MP2 force field cannot nicely predict the DFT-energies computed in the periodic system. The energies of $\mathrm{CO}_{2}$ configurations located near the center of the pores are systemically overestimated. In addition, the minimum energy configuration that is predicted by this force field has a correct energy, but gives a different orientation than the one predicted by DFT. DFT predicts that the minimum energy configuration of $\mathrm{O}=\mathrm{C}=\mathrm{O}$ has an angle of $\sim 80^{\circ}$ with respect to the axis parallel to the channel, whereas the corresponding angle predicted by MP2 force field is significantly smaller $\left(<60^{\circ}\right)$. Predicting this angle correctly is significantly important to reproduce the NMR measured lineshape induced by $\mathrm{CO}_{2}$ dynamics (see next section). These results suggest that the previously adopted clusters may have been too small to capture the range of possible interactions in the extended structure.

To test the robustness of our methodology, we replace the $\mathrm{C}_{6}$ coefficients with values adopted from Grimme's DFT-D2 method $^{29}$ for the dispersive interactions between framework atoms and $\mathrm{O}\left(\mathrm{CO}_{2}\right)$ (i.e., approached pairs); the other interactions were kept the same as in model 1. This force field is denoted as model 2. Figure 4 shows that the predicted isotherm of this model is nearly identical to the isotherm predicted by model 1, suggesting that our methodology is not very sensitive to the choice of initial $\mathrm{C}_{6}$ coefficients for the approached pairs.

To test the sensitivity of our approach to the details of the $\mathrm{CO}_{2}$ model, we further replace the EPM2 model by the TraPPE model for $\mathrm{CO}_{2}$, which we refer to as model 3. The calculated isotherm is again very similar to the ones obtained from models 1 and 2, although a slightly different loading at higher-pressure region is observed. Compared to the TraPPE model, the EPM2 has similar Lennard-Jones parameters but different partial atomic charges (i.e., $-0.35 e$ vs $-0.325 e$ for the oxygen of $\mathrm{CO}_{2}$, respectively). The similarity of the isotherms predicted by models 1 and 3 demonstrates that the differences in electrostatic energies can be taken into account correctly by adjusting the pairwise repulsive energies.

Although the predicted isotherms of these three models show generally good agreement with the experimental isotherms, the calculated Henry coefficients are about 3 times smaller than the experimental values. To understand these differences, we introduce a rotational motion of $\mathrm{CO}_{2}$ as shown in the inset of Figure 5. Figure 5 elucidates that model 1 fails to capture the DFT energy as a function of rotational angle. This model underestimates the interaction energies if $\mathrm{CO}_{2}$ rotates toward the linker. The pairwise parameters between $\mathrm{C}\left(\mathrm{CO}_{2}\right)$ and framework atoms are not optimized and, as these three models have similar parameters for $\mathrm{C}\left(\mathrm{CO}_{2}\right)$, similar deviations can be expected for the other models. A further improvement of the force field might be obtained by refining the parameters between $\mathrm{C}\left(\mathrm{CO}_{2}\right)$ and the framework. Unfortunately, our methodology is not applicable to the middle atom of a linear molecule (i.e., unapproachable pairs). To obtain some insights

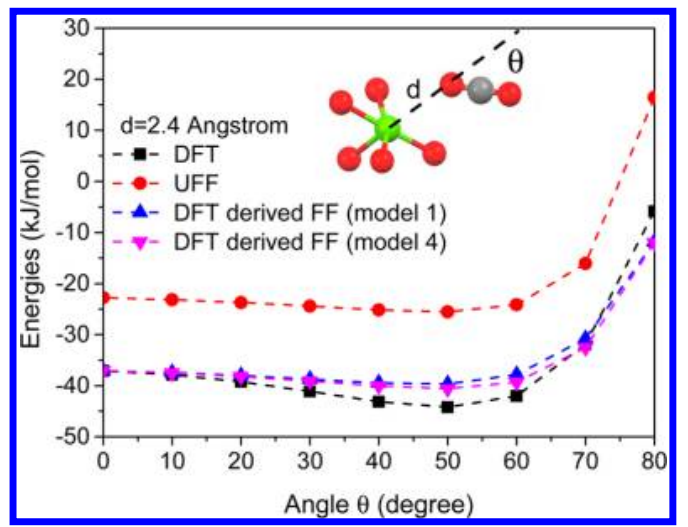

Figure 5. Comparison between the DFT and force-field interaction energies as a function of the angle $\theta$. This angle gives the orientation of $\mathrm{O}=\mathrm{C}=\mathrm{O}$ with respect to the vector of $\mathrm{Mg}-\mathrm{CO}_{2}$ approaching path (see the inset of figure). The $\mathrm{O}-\mathrm{Mg}$ distance, $d$, is set to $d=2.4 \AA$. Black closed squares are the DFT reference energies and the other symbols are the corresponding energies calculated by different force fields.

in the role of the $\mathrm{C}\left(\mathrm{CO}_{2}\right)$ interactions, we replace the parameters for the framework- $\mathrm{C}\left(\mathrm{CO}_{2}\right)$ interactions in model 2 with the $\mathrm{C}_{6}$ coefficients from DFT-D2 method. As these coefficients were optimized from quantum calculations, they might give a more consistent result with the rest of the force field. Given that DFT-D2 method does not provide information on the repulsive part of the potential, we assume that the sigma parameters used in the Lennard-Jones potential has the value equal to the summation of atomic van der Waal radii. The obtained set of parameters is denoted as model 4 . Figure 5 shows a small improvement for this model. Additionally, the Boltzmann-weighted mean deviation of model 4 for the set of 1200 random configurations (see SI Figure S4) is $0.76 \mathrm{~kJ} / \mathrm{mol}$, which is smaller than that of model 1. These improvements are also reflected in the predicted isotherm, which has better agreement with the experimental data at low-pressure region. In the high-pressure region, the loading is overestimated, which might be due to our assumptions of the repulsive parameters between framework$\mathrm{C}\left(\mathrm{CO}_{2}\right)$. The overestimation of the predicted energies can also be seen for a small portion of the randomly generated configurations (see SI Figure S4) at intermediate adsorption energies (i.e., $-25-0 \mathrm{~kJ} / \mathrm{mol}$ ). It should be noted that all of these 4 models has their corresponding global scaling factor, $S_{g}$, to be further fine-tuned to minimize difference between the energies computed by the force fields and by DFT along all the paths.

Furthermore, in Figure 6, we compare the binding geometry computed by model 4 with the corresponding geometry obtained from DFT calculations. Table 1 gives a quantitative comparison of the distances between $\mathrm{CO}_{2}$ and a few selected framework atoms. The comparison clearly demonstrates that model 4 nicely reproduces the DFT binding geometry. It is also important to note that the predicted DFT binding geometry agrees very well with the experimental binding geometry, as obtained from the neutron powder diffraction experiment at 20 $\mathrm{K}$ by Queen et al. ${ }^{19}$

Transport Properties. At this point, it is instructive to apply our derived force field to study the dynamic properties of $\mathrm{CO}_{2}$ inside Mg-MOF-74. In our previous study, we used the UFF force field to predict the experimentally measured ${ }^{13} \mathrm{C}$ NMR 


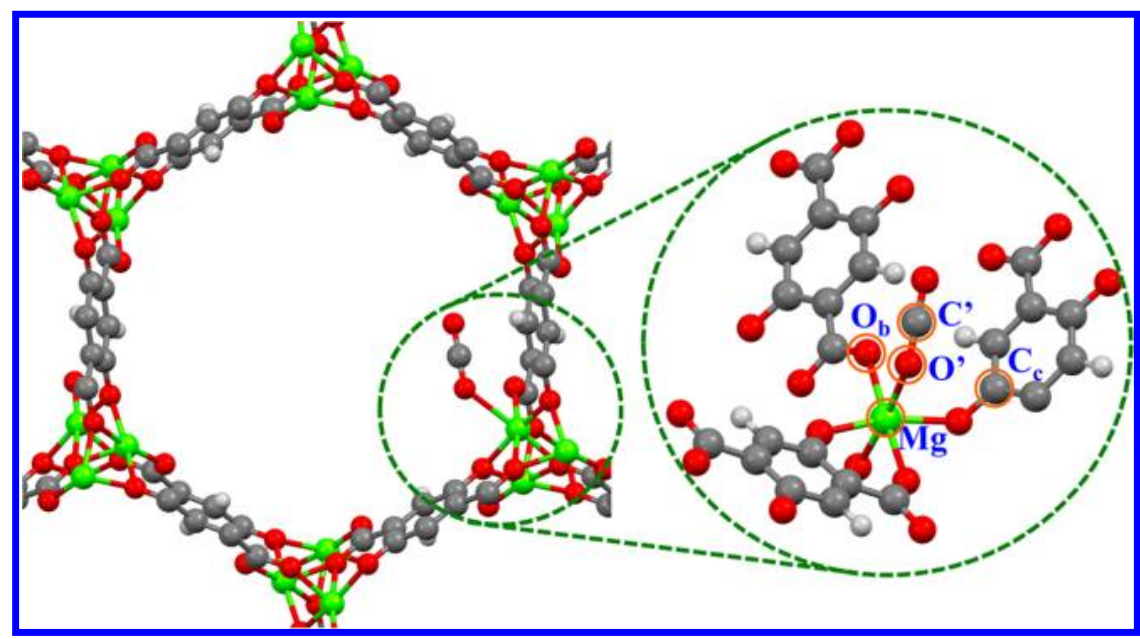

Figure 6. DFT Binding geometry of a single $\mathrm{CO}_{2}$ molecule adsorbed inside Mg-MOF-74. The figure represents the material as balls and sticks (Mg, green; $\mathrm{O}$, red; $\mathrm{C}$, gray; $\mathrm{H}$, white). The inset amplifies the binding region.

Table 1. Comparison of Binding Geometries Obtained from DFT-Derived Force Field, DFT, and Neutron Power Diffraction (NPD) ${ }^{a}$

\begin{tabular}{lccc} 
& \multicolumn{3}{c}{ distance $(\AA)$} \\
\cline { 2 - 4 } \multicolumn{1}{c}{ source } & $\mathrm{O}^{\prime}-\mathrm{Mg}$ & $\mathrm{C}^{\prime}-\mathrm{O}_{\mathrm{b}}$ & $\mathrm{C}^{\prime}-\mathrm{C}_{\mathrm{c}}$ \\
DFT & 2.40 & 2.94 & 3.37 \\
DFT-derived force field & 2.42 & 2.94 & 3.25 \\
$\mathrm{NPD}^{b}$ (Queen et al.) & 2.39 & 3.10 & 3.07
\end{tabular}

${ }^{a_{T}}$ The atomic labels used in this table can be found in Figure 6 of the manuscript. ${ }^{b}$ At $20 \mathrm{~K}$ and $0.25 \mathrm{CO}_{2} / \mathrm{Mg}$.

line shapes. ${ }^{30}$ The uniaxial rotational-like NMR patterns were properly explained in terms of a hopping motion of $\mathrm{CO}_{2}$ along the $X-Y$ plane. ${ }^{30}$ The UFF force field does give a reasonable prediction of the binding orientation and, hence, correctly predicts the equivalent rotational angle resulted from the $\mathrm{CO}_{2}$ hopping from one metal site to another. However, as the binding energies are predicted incorrectly by UFF, we were unable to predict the correct transition temperature of experimental NMR patterns from fixation to hopping motion. ${ }^{31}$

An important test of our new force field is whether we now can correctly predict this transition temperature. Accordingly, molecular dynamics simulations are performed with model 4, and a collection of $\mathrm{MD}$ trajectories is analyzed. Figure 7 shows that the hopping rate along the $X-Y$ plane becomes nonzero above $150 \mathrm{~K}$, which is exactly the temperature where experimentally measured pattern shows the intermediate motion. $^{31}$ Furthermore, for the activation energies of the hopping motions along the $X-Y$ plane and Z-direction, we obtain 10.7 and $11.4 \mathrm{~kJ} / \mathrm{mol}$, respectively, from our calculations. These activation energies are in good agreement with the experimental value, which is the average of these two activation energies (i.e., $10.0 \mathrm{~kJ} / \mathrm{mol}$ ). Additionally, our force field predicts a slightly higher barrier of the hopping along the $Z$-direction than along the $X-Y$ plane. The result is consistent with the free energy barrier calculation performed by Canepa et al. using the ab initio nudged elastic band (NEB) method. ${ }^{32}$

$\mathrm{CO}_{2}$ Adsorption in Zn-MOF-74. The transferability of the methodology is evaluated by studying $\mathrm{CO}_{2}$ inside $\mathrm{Zn}$-MOF-74. Compared to the Mg-MOF-74 case, the main differences of the DFT energies are observed along the path toward the metal center. All other paths give very similar energies as for the $\mathrm{Mg}$ -

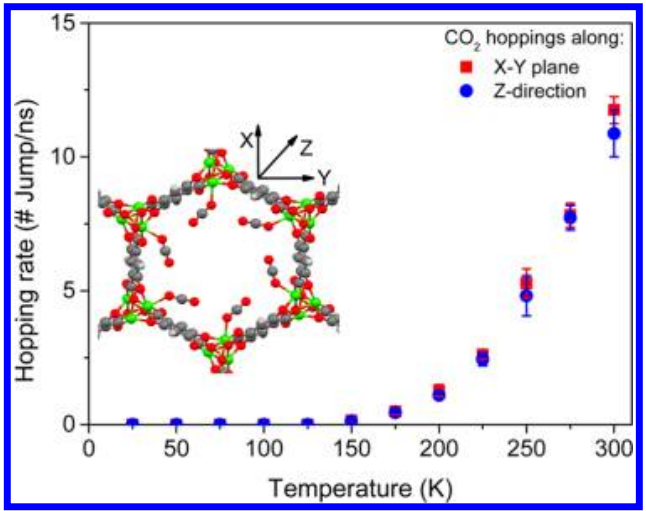

Figure 7. Hopping rate (number of jumps per ns) of $\mathrm{CO}_{2}$ along the $X-Y$ plane (red squares) and Z-direction (blue circles) as a function of temperature $(\mathrm{K})$. The inset illustrates the pore topology as well as the coordinates system. The Z-direction corresponds to the crystallographic $c$-axis.

MOF-74 case. In this regard, the $\mathrm{CO}_{2}-\mathrm{Zn}$ approaching path is the only path required to be reoptimized. We develop the force field for Zn-MOF-74 upon the model 4 for Mg-MOF-74. Figure S5 of the SI shows that the obtained force fields with only the metal path to be reparametrized can indeed reproduce the DFT energies along all other paths (i.e., $\mathrm{O}$ and $\mathrm{C}$ paths). Figure 8 shows that, for $\mathrm{Zn-MOF-74,} \mathrm{our} \mathrm{predicted} \mathrm{isotherm} \mathrm{is}$ also in good agreement with the experimentally scaled one. ${ }^{33}$ Given that we can obtain such a good agreement by only reparameterizing the metal-guest interactions, indicating that the parameters obtained in the Mg-MOF-74 are transferable to the $\mathrm{Zn}$-MOF-74 as well as the method itself.

$\mathrm{H}_{2} \mathrm{O}$ Adsorption in Mg-MOF-74. We also apply the methodology to $\mathrm{H}_{2} \mathrm{O}$ that is a major component, next to $\mathrm{N}_{2}$ and $\mathrm{CO}_{2}$, of flue gases. For water we use the TIP4P-Ew model. ${ }^{34}$ According to the TIP4P-Ew model, there is no nonelectrostatic interactions between framework atoms and $\mathrm{H}\left(\mathrm{H}_{2} \mathrm{O}\right)$, and therefore $\mathrm{H}\left(\mathrm{H}_{2} \mathrm{O}\right)$ is not considered explicitly for paths determinations. Our study shows that, however, the resulting model without considering the nonelectrostatic interactions between framework- $\mathrm{H}\left(\mathrm{H}_{2} \mathrm{O}\right.$ ) (model A) leads to unrealistic energies of some configurations. This problem can be further corrected by introducing $\mathrm{H}\left(\mathrm{H}_{2} \mathrm{O}\right)$ approaching paths explicitly into parametrization loops (model B). During the 


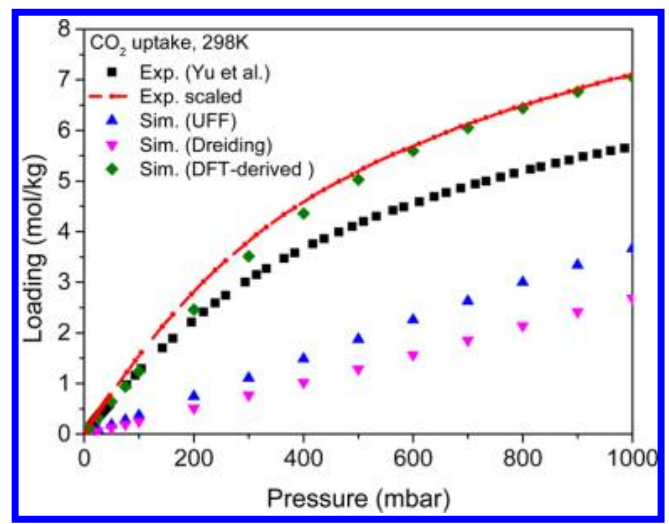

Figure 8. Comparison between the experimental and simulated isotherms of $\mathrm{CO}_{2}$ inside $\mathrm{Zn}-\mathrm{MOF}-74$ at $298 \mathrm{~K}$. The experimental data of Yu et al. ${ }^{33}$ and the corresponding scaled isotherm (assume only $80 \%$ of material are accessible, which is the same value we have used for Mg-MOF-74) are shown by the closed black squares and red close circle with dashed line, respectively. The symbols are the isotherms from the GCMC simulations as predicted by the different models.

development of water models, sufficiently large global scaling factors, $S_{g}$, are chosen and have not been further optimized.

Similar to $\mathrm{CO}_{2}$, a total of 8 paths (i.e., $\mathrm{Mg}, \mathrm{O}_{a}, \mathrm{O}_{b}, \mathrm{O}_{\mathcal{c}} \mathrm{C}_{a}, \mathrm{C}_{b}$, $\mathrm{C}_{c}$, and $\left.\mathrm{C}_{d}\right)$ are determined in which the $\mathrm{O}\left(\mathrm{H}_{2} \mathrm{O}\right)$ is approaching toward the different framework atom-types. We find that the path targeting the $\mathrm{O}_{a}$ atom does not give a satisfactory fitting of the DFT-energies. The $\mathrm{O}_{\mathrm{a}}$ atom is more embedded in the framework compared to other atom-types, and therefore, the repulsion between the approached pair of $\mathrm{O}_{a}$ and $\mathrm{O}\left(\mathrm{H}_{2} \mathrm{O}\right)$ has a much smaller contribution to the net repulsion compared to the other atom-types. The reason is that the repulsion from the neighbor framework atoms prevents the $\mathrm{O}\left(\mathrm{H}_{2} \mathrm{O}\right)$ from probing the short-range repulsion with $\mathrm{O}_{\mathrm{a}}$. Accordingly, the corresponding pairwise repulsive parameters will be far less sensitive to the DFT-decomposed repulsion and cannot be obtained accurately. Such a poorly defined path degrades the stability of the parametrization loop. At the same time because of this effect the correction for the short-range repulsion of this pair is less important and can therefore be omitted from the optimization loop. Without the $\mathrm{O}_{a}$ path, the DFT energies along all other 7 approaching paths are nicely fitted as shown in Figure 9. We denote the set of parameters as model A.
To test the reliability of model $\mathrm{A}$, we also generate a set of 2000 random $\mathrm{H}_{2} \mathrm{O}$ configurations in the accessible pores of this system. The comparison between the DFT and force-field energies for these configurations is shown in Figure 10a. Compared to the UFF, our new force field shows a much better agreement with the DFT energies. However, model A highly overestimates the interaction energies for some configurations. For example, for one of these configurations the energy calculated from the DFT is $-15 \mathrm{~kJ} / \mathrm{mol}$ while model A gives $-55 \mathrm{~kJ} / \mathrm{mol}$. Detailed inspection of these configurations shows that the hydrogen site in the TIP4P-Ew model causes these differences. The $\mathrm{H}\left(\mathrm{H}_{2} \mathrm{O}\right)$ site has a positive partial charge, but the site does not include repulsive interaction, which artificially enables $\mathrm{H}\left(\mathrm{H}_{2} \mathrm{O}\right)$ getting close to negatively charged framework atoms and thus gain favorable energies. To correct the interaction between $\mathrm{H}\left(\mathrm{H}_{2} \mathrm{O}\right)$-framework, 2 hydrogen-approaching paths (i.e., $\mathrm{O}_{b}-\mathrm{H}$ and $\mathrm{C}_{b}-\mathrm{H}$ paths) are generated and included in the self-consistent parametrization loops. The fitted parameters from the $\mathrm{O}_{b}-\mathrm{H}$ and $\mathrm{C}_{b}-\mathrm{H}$ paths are applied to other framework atom-types with the same element type. The resulting force field is denoted as model $\mathrm{B}$.

Figure 11 compares the DFT and force-field energies along these two hydrogen paths. Model B perfectly reproduces the energies along the paths, whereas model A highly overestimates the interaction. It is intriguing to see that the UFF force field does not seriously overestimate the energies along these paths although there is no physical repulsion applied on the $\mathrm{H}\left(\mathrm{H}_{2} \mathrm{O}\right)$. The overestimation of the repulsive interaction in the $\mathrm{O}\left(\mathrm{H}_{2} \mathrm{O}\right)$-framework protects the hydrogen atoms of water and, hence, avoids this issue. Figure 10a illustrates that the energies computed by model B are in much better agreement with the DFT energies compared to the previous model. Moreover, another set of 1000 configurations is chosen to sample the region near the open-metal sites in order to concentrate on water binding region. Figure $10 \mathrm{~b}$ again illustrates that the model B nicely reproduces the DFT energies and shows huge improvement compared to the UFF. Similarly, we computed the Boltzmann-weighted mean deviations at 300 $\mathrm{K}$ for the UFF and model B with the set of selected 1000 configurations, and the values are 47.70 and $1.07 \mathrm{~kJ} / \mathrm{mol}$, respectively.

In Figure 12a, we compare the water isotherms predicted by the model $\mathrm{B}$ and the UFF with the corresponding experimental isotherms reported in the literature. ${ }^{35-37}$ The model $B$
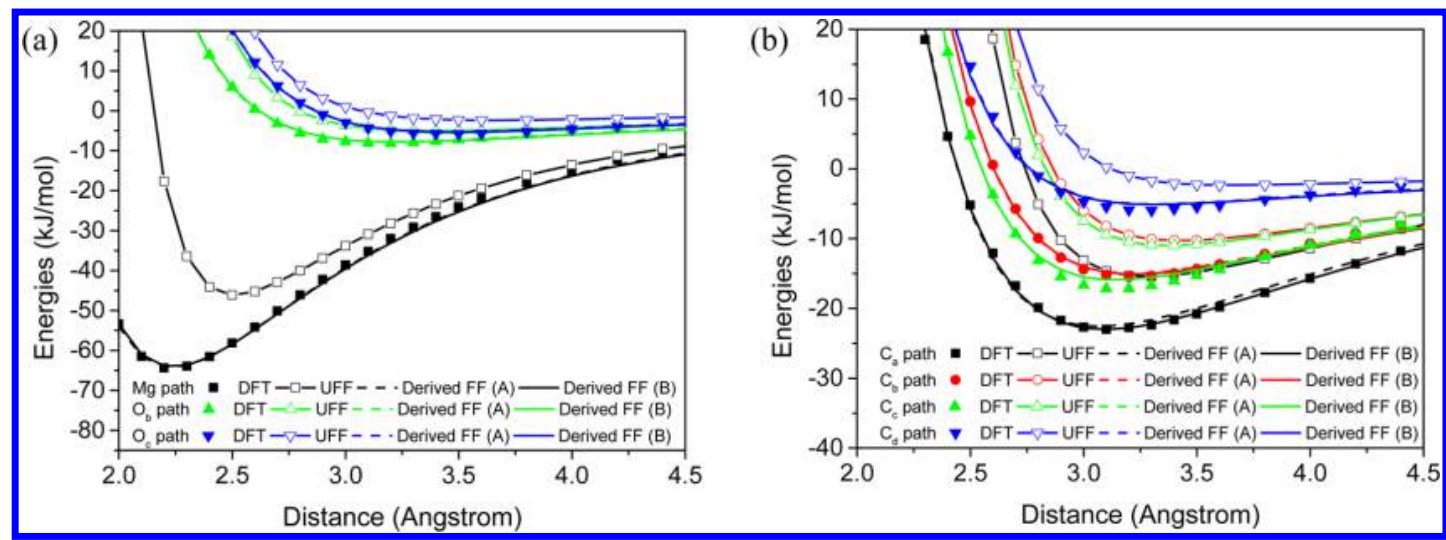

Figure 9. Comparison of $\mathrm{H}_{2} \mathrm{O}$-framework interaction energies as a function of distance as obtained from the DFT calculations (closed symbols) with ones computed from the UFF (open symbols with solid lines) and the DFT-derived force field (dashed and solid lines for model A and B, respectively) along (a) $\mathrm{Mg}$ and $\mathrm{O}$ and (b) $\mathrm{C}$ paths. 

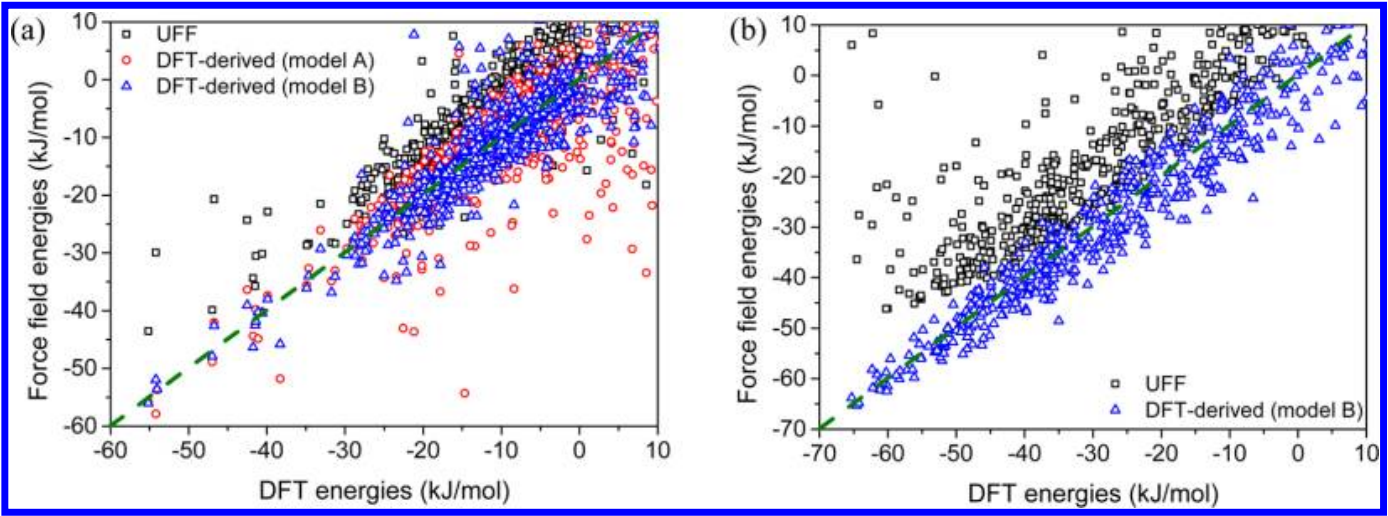

Figure 10. Comparison of the interaction energies obtained from the DFT calculations with the ones computed from the UFF (open black squares), the DFT-derived force field (model A, open red circles), and the DFT-derived force field (model B, open upper-triangles) for a set of randomly selected (a) $2000 \mathrm{H}_{2} \mathrm{O}$ configurations in the accessible pore volume and (b) $1000 \mathrm{H}_{2} \mathrm{O}$ configurations located near the open metal sites inside $\mathrm{Mg}$ MOF-74. The green-dashed line indicates the location where the energies computed by DFT and by force fields are identical.

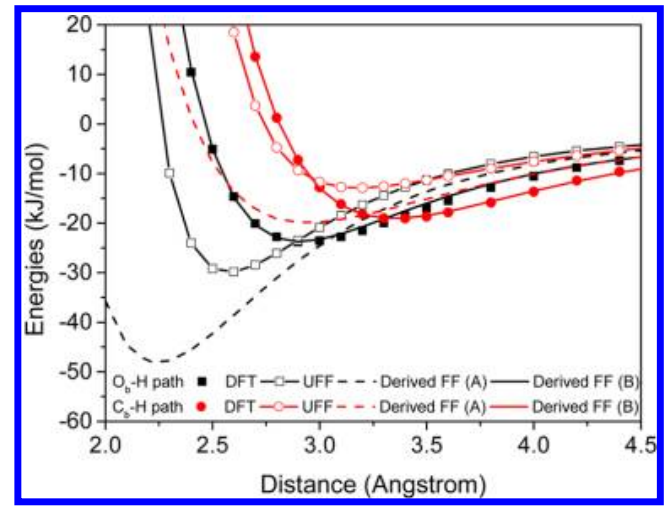

Figure 11. Comparison of the $\mathrm{H}_{2} \mathrm{O}$-framework interaction energies as a function of distance obtained from the DFT calculations (closed symbols) with ones computed from the UFF (open symbols with solid lines) and the DFT-derived force fields (dashed and solid lines for model $\mathrm{A}$ and $\mathrm{B}$, respectively) for $\mathrm{O}_{\mathrm{b}}-\mathrm{H}$ (black) and $\mathrm{C}_{\mathrm{b}}-\mathrm{H}$ (red) paths.

isotherm agrees much better with the experimental data of Yang et al. (C) ${ }^{35}$ compared to the UFF isotherm at the lowpressure region. At the higher-pressure region (i.e., $P=0.1-1$
$\mathrm{kPa}$ ); however, both the model $\mathrm{B}$ and UFF predict a much higher loading than the experimental measurement. The water adsorption isotherms in Mg-MOF-74 reported in the literature have, however, a wide range of uptake values. The experimental data of Schoenecker et al. ${ }^{36}$ has comparable uptake at higherpressure region with the one obtained by Yang et al. (C), whereas Glover et al. ${ }^{37}$ obtain up to $70 \%$ lower adsorption. Moreover, Yang et al. show different adsorption behaviors with the Mg-MOF-74 samples synthesized from different methods (i.e., (C) vs (S)). These large variations illustrate the difficulties in measuring the water uptake. These differences make it difficult to make quantitative statements on the reliability of our predicted isotherms at higher-pressure region. Same procedure (i.e., model B) is also applied to generate force field of $\mathrm{H}_{2} \mathrm{O}$ in $\mathrm{Zn}-\mathrm{MOF}-74$, and the resulted isotherm is given in Figure $12 \mathrm{~b}$. Interestingly, we find different adsorption mechanisms of $\mathrm{H}_{2} \mathrm{O}$ molecules adsorbed inside Mg-and Zn-MOF-74. ZnMOF-74 shows a much sharper transition, resembling capillary condensation, in the water adsorption isotherm as compared to Mg-MOF-74. To understand these differences, we partitioned the average energies per $\mathrm{H}_{2} \mathrm{O}$ molecule into framework-guest and guest-guest interactions (See Figure 13). The results show that, because of the strong $\mathrm{H}_{2} \mathrm{O}-$ metal interactions in $\mathrm{Mg}$ -
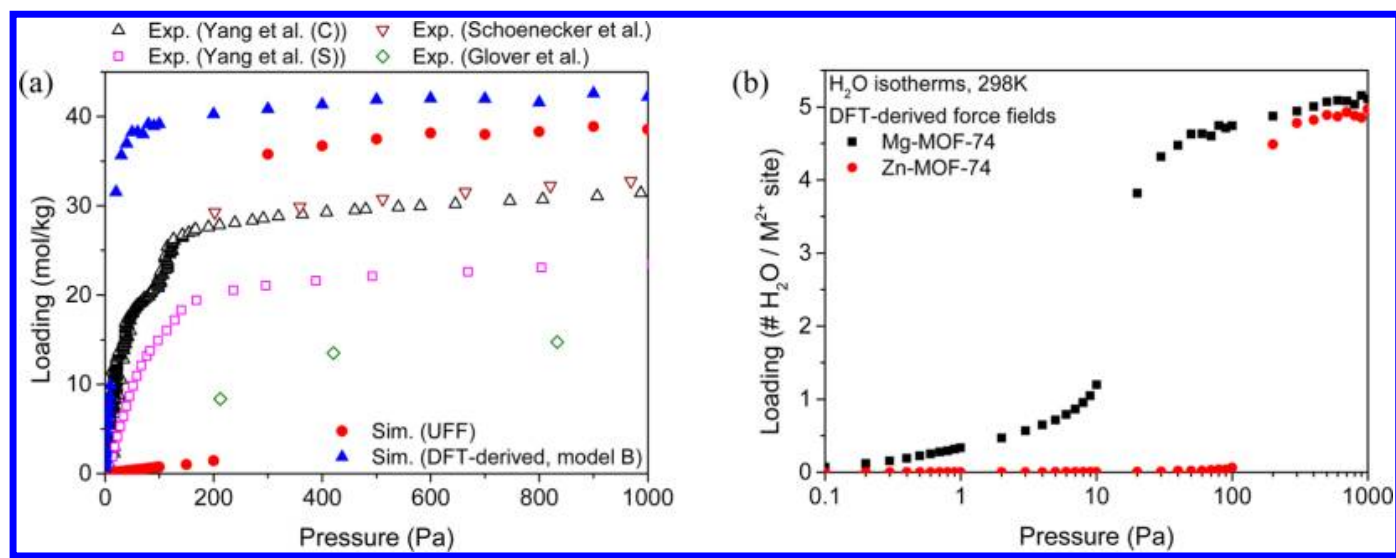

Figure 12. (a) Comparison between the experimental and simulated isotherms for $\mathrm{H}_{2} \mathrm{O}$ inside Mg-MOF-74 at $298 \mathrm{~K}^{35-37}$ The experimental isotherms are shown by the open symbols. The closed red circles and blue closed upper-triangles represent the computed isotherms with the UFF and the DFT-derived force field (model B), respectively. (b) Calculated $\mathrm{H}_{2} \mathrm{O}$ adsorption isotherms at $298 \mathrm{~K}$ inside Mg-MOF-74 (closed black squares) and Zn-MOF-74 (closed red squares). In these calculations, DFT-derived force fields (model B; explicitly takes $\mathrm{H}\left(\mathrm{H}_{2} \mathrm{O}\right)$ paths into the parametrization loops) are used. 


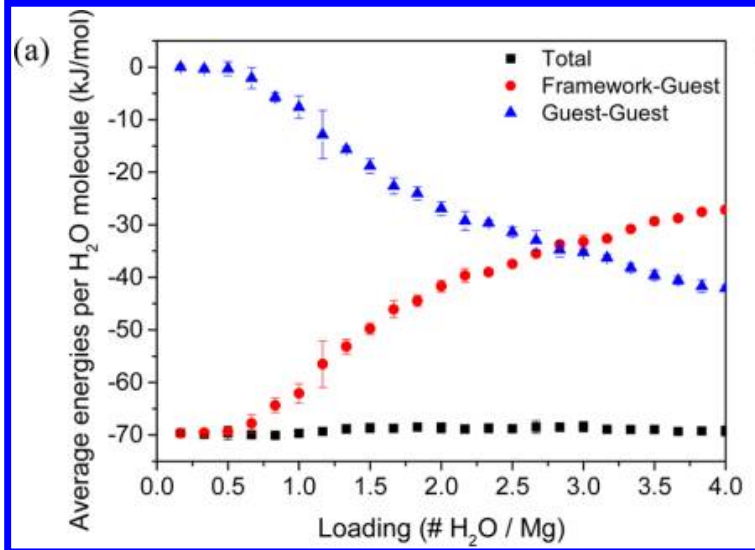

(b)

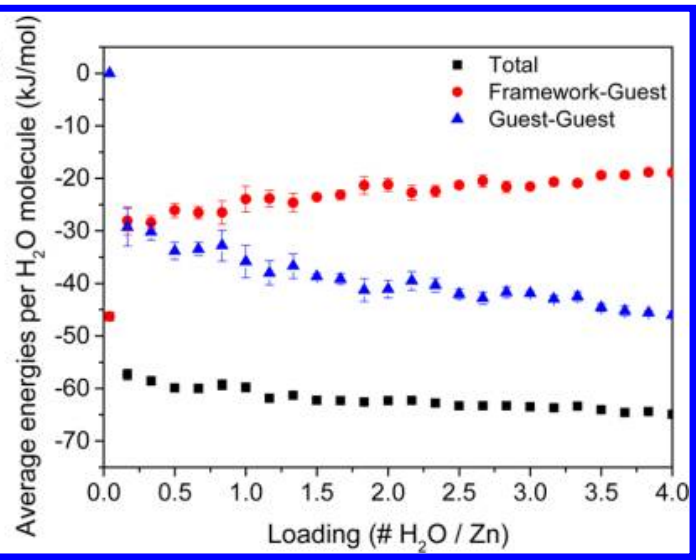

Figure 13. Average energies per $\mathrm{H}_{2} \mathrm{O}$ molecule $(\mathrm{kJ} / \mathrm{mol})$ as a function of loading $\left(\# \mathrm{H}_{2} \mathrm{O} /\right.$ metal) adsorbed inside (a) Mg-MOF-74 and (b) ZnMOF-74. To illustrate the energy contributions of framework-guest and guest-guest to the total energies, the total energies and these two components are plotted separately. In these calculations, DFT-derived force fields (model B; explicitly takes $\mathrm{H}\left(\mathrm{H}_{2} \mathrm{O}\right)$ paths into the parametrization loops) are used.

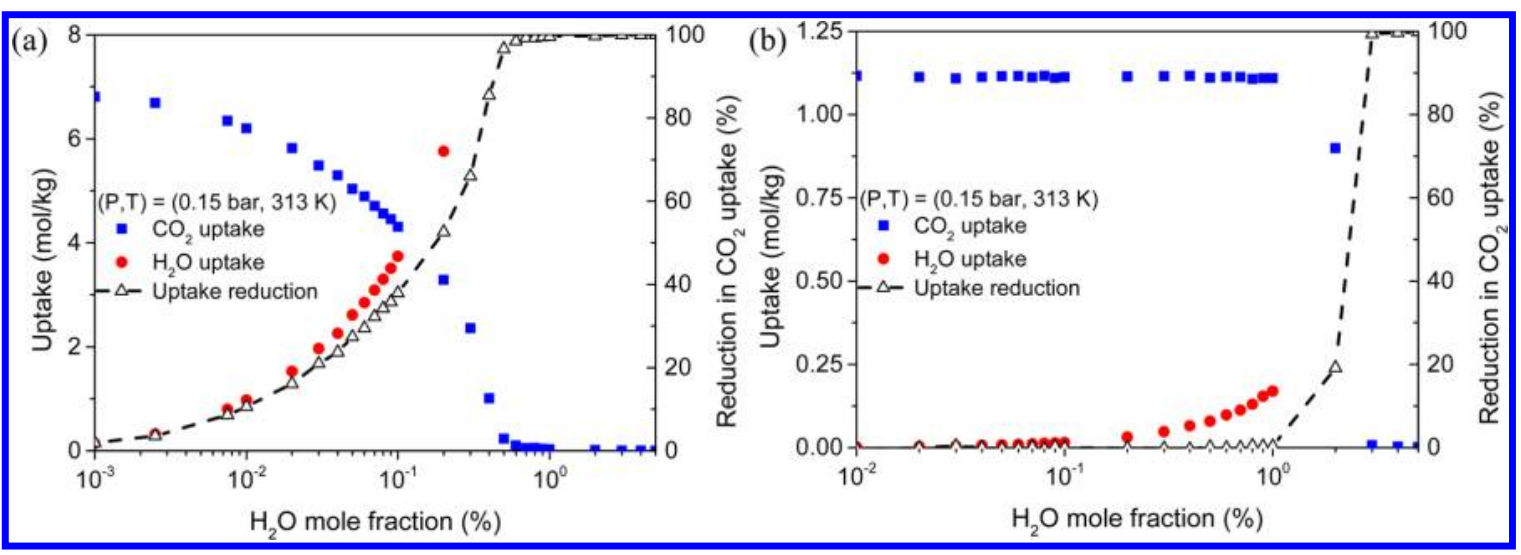

Figure 14. Binary mixture inside (a) Mg-MOF-74 and (b) Zn-MOF-74. Left $y$-axis: simulated binary mixture uptake of $\mathrm{CO}_{2}$ (red closed circles) and $\mathrm{H}_{2} \mathrm{O}$ (closed blue squares) as a function of $\mathrm{H}_{2} \mathrm{O}$ mole fraction at total pressure of 0.15 bar and temperature of $313 \mathrm{~K}$. Right $y$-axis: the reduction of $\mathrm{CO}_{2}$ uptake (\%) as a function of $\mathrm{H}_{2} \mathrm{O}$ mole fraction. The definition of reduction is the ratio of $\mathrm{CO}_{2}$ uptake under the mixture condition to the pure component $\mathrm{CO}_{2}$ uptake at $(P, T)=(0.15 \mathrm{bar}, 313 \mathrm{~K})$.

MOF-74, the $\mathrm{H}_{2} \mathrm{O}$ molecules tend to fully occupy all openmetal sites before condensation. This is consistent with the shape of $\mathrm{H}_{2} \mathrm{O}$ isotherm in Mg-MOF-74, which has the condensation of $\mathrm{H}_{2} \mathrm{O}$ molecules at higher loadings, that is, beyond one $\mathrm{H}_{2} \mathrm{O}$ molecule per metal site. In contrast, for $\mathrm{Zn}$ MOF-74, the interactions between $\mathrm{H}_{2} \mathrm{O}$ and metal sites are much weaker. As a consequence, once there is one $\mathrm{H}_{2} \mathrm{O}$ molecule adsorbed on an open metal site, for the next $\mathrm{H}_{2} \mathrm{O}$ molecule it would be energetically more favorable to be adsorbed in proximity to the first $\mathrm{H}_{2} \mathrm{O}$ molecule due to the strong $\mathrm{H}_{2} \mathrm{O}-\mathrm{H}_{2} \mathrm{O}$ interactions. As we can clearly see from Figure 13(b), the strong guest-guest interactions essentially introduce a more negative average adsorption energies of $\mathrm{H}_{2} \mathrm{O}$ molecules compared to infinite dilution conditions.

$\mathrm{CO}_{2} / \mathrm{H}_{2} \mathrm{O}$ Mixture Adsorption in M-MOF-74 ( $\mathrm{M}=\mathrm{Mg}$ and $\mathrm{Zn})$. The presence of water has been regarded as one of the key problems for the application of MOFs with open metal sites in CCS. With our novel force fields for $\mathrm{CO}_{2}$ and $\mathrm{H}_{2} \mathrm{O}$, we are in a position to make quantitative prediction on the effect of water on the uptake of $\mathrm{CO}_{2}$ at the typical mixture condition of flue gases for both Mg-MOF-74 and Zn-MOF-74. To the best of our knowledge, there are no experimental mixture data available on these systems.
Since nitrogen has a much weaker binding strength inside $\mathrm{M}$ MOF-74 compared to the other main components (i.e., $\mathrm{CO}_{2}$ and $\mathrm{H}_{2} \mathrm{O}$ ) of the flue gases emitted from a coal-fired power plant, ${ }^{38,39}$ we can focus on the binary mixture of $\mathrm{CO}_{2}$ and $\mathrm{H}_{2} \mathrm{O}$ and simply assume that $\mathrm{N}_{2}$ does not adsorb at these conditions. Our simplified flue gases are at total pressure of 0.15 bar and at temperature of $313 \mathrm{~K}$ with a range of $\mathrm{H}_{2} \mathrm{O}$ mole fraction varying from $0.001 \%$ to $5 \%$. The mixture GCMC simulations are performed to predict the uptake at each specific composition. To quantify the effect of water on the $\mathrm{CO}_{2}$ uptake performance, we introduce a reduction factor. This factor is defined as the ratio of $\mathrm{CO}_{2}$ uptake in the presence of water vapor to pure component $\mathrm{CO}_{2}$ uptake at $(P, T)$ equal to (0.15 bar, $313 \mathrm{~K})$.

For the mixture inside Mg-MOF-74, the simulated uptake and the reduction factor as a function of $\mathrm{H}_{2} \mathrm{O}$ composition is shown in Figure 14(a). Surprisingly, the result shows that the reduction of $\mathrm{CO}_{2}$ reaches $50 \%$ in the presence of $0.2 \%$ water. At $0.5 \%$, the $\mathrm{CO}_{2}$ uptake is essentially equal to zero, which indicates that the material has no capability of adsorbing carbon dioxide. A detailed investigation shows that the computed binding energy of $\mathrm{H}_{2} \mathrm{O}$ is strong (i.e., $\sim-70 \mathrm{~kJ} / \mathrm{mol}$ ), and the difference in the isosteric heat of adsorption between $\mathrm{H}_{2} \mathrm{O}$ and $\mathrm{CO}_{2}$ is large (i.e., $>20 \mathrm{~kJ} / \mathrm{mol}$ ). In this regard, $\mathrm{H}_{2} \mathrm{O}$ molecules 
are expected to adsorb on the open metal sites very strongly, and in a much more energetically favorable way than $\mathrm{CO}_{2}$. Accordingly, one would need very dry flue gases for Mg-MOF74. Otherwise the separation performance will be degraded enormously.

It is interesting to compare the effect of water on adsorption of $\mathrm{CO}_{2}$ in $\mathrm{Zn}-\mathrm{MOF}-74$. Compared to the Mg-MOF-74, given that the binding strength of water is much weaker, $\mathrm{Zn}-\mathrm{MOF}-74$ is less sensitive to the presence of trace amounts of water vapor. Our calculations show that there is nearly no degradation in the $\mathrm{CO}_{2}$ uptake before the occurrence of water condensation inside the Zn-MOF-74 (i.e., 2\%, see Figure 14(b)).

\section{CONCLUDING REMARKS}

In summary, we have developed a new method to derive accurate (nonpolarizable) force fields from periodic DFT calculations. The characteristic of this method is that we only calculate DFT energies on specific paths that are most sensitive to their corresponding pairwise interactions between approached pairs. Compared to methods that use genetic algorithms, this reduces the number of required $a b$ initio calculations. In addition, we use an energy decomposition scheme in combination with a self-consistent optimization loop, which allows us to fit only two parameters at the same time. Our method therefore significantly reduces the computational cost to develop ab initio force fields and is relatively simple to be implemented. Part of the gain in efficiency is due to the fact that our approach uses one of the common force fields (e.g., UFF) as a starting point and aims to correct these. We have also tested the robustness of this methodology by showing that the predicted adsorption properties of the derived force fields are insensitive to the choice of the initial force fields. We anticipate that this methodology can be generally applicable for metal-organic frameworks or even some other classes of porous materials. However, the method might have limitations in those structures that possess too small channels or pores to properly generate approaching paths.

In this work, we have demonstrated that these new force fields yield accurate estimates of the $\mathrm{CO}_{2}$ adsorption properties inside Mg-MOF-74. We also studied the dynamics of the adsorbed $\mathrm{CO}_{2}$ molecules in Mg-MOF-74. Both the transition temperature from fixation to hopping motion and the activation energies of $\mathrm{CO}_{2}$ hopping motion nicely match the experimental observations. To test transferability of the methodology, we have also studied $\mathrm{CO}_{2}$ adsorbed inside Zn-MOF-74, and the predicted isotherm is in excellent agreement with the experimental data.

Moreover, we have developed a force field for $\mathrm{H}_{2} \mathrm{O}$. This water force field gives a reasonable prediction on the water adsorption inside Mg-MOF-74 and provides significant improvements compared to the prediction made by the UFF force field. Additionally, very different $\mathrm{H}_{2} \mathrm{O}$ adsorption mechanisms between Mg-MOF-74 and $\mathrm{Zn-MOF-74}$ are found due to the difference in the $\mathrm{H}_{2} \mathrm{O}$-metal binding strength. Finally, with these new force fields, we make further predictions on the $\mathrm{CO}_{2} / \mathrm{H}_{2} \mathrm{O}$ mixture adsorption properties, and the results show that the performance of both $\mathrm{Mg}$ - and $\mathrm{Zn}-\mathrm{MOF}-74$ to uptake $\mathrm{CO}_{2}$ can be degraded enormously by the presence of water. Compared to Mg-MOF-74, we find that Zn-MOF-74, however, can tolerate roughly four times higher in water concentration without sacrificing considerable ability of adsorbing $\mathrm{CO}_{2}$.

\section{ASSOCIATED CONTENT}

\section{Supporting Information}

Additional figures as described in the text; detailed force field parameters derived in this study. This material is available free of charge via the Internet at http://pubs.acs.org.

\section{AUTHOR INFORMATION}

\section{Corresponding Authors}

*E-mail: lichianglin@berkeley.edu.

*E-mail: kyuholee@lbl.gov.

\section{Author Contributions}

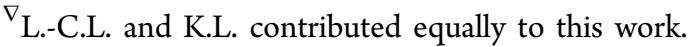

\section{Notes}

The authors declare no competing financial interest.

\section{ACKNOWLEDGMENTS}

We gratefully thank Prof. Jihan Kim at Korea Advanced Institute of Science and Technology (KAIST) for providing help with code modifications to facilitate the development of this methodology. This research was supported by the U.S. Department of Energy, Office of Basic Energy Sciences, Division of Chemical Sciences, Geosciences, and Biosciences under award DE-FG02-12ER16362. Work at the Molecular Foundry was supported by the Office of Science, Office of Basic Energy Sciences, of the U.S. Department of Energy under Contract No. DE-AC02-05CH11231. L.-C.L. acknowledges financial support from a Chevron Fellowship. This research used resources of the National Energy Research Scientific Computing Center (NERSC), which is supported by the Office of Science of the U.S. Department of Energy under Contract No. DE-AC02-05CH11231.

\section{REFERENCES}

(1) Furukawa, H.; Cordova, K. E.; O’Keeffe, M.; Yaghi, O. M. The Chemistry and Applications of Metal-Organic Frameworks. Science 2013, 341, 1230444.

(2) D'Alessandro, D. M.; Smit, B.; Long, J. R. Carbon Dioxide Capture: Prospects for New Materials. Angew. Chem., Int. Ed. 2010, 49, $6058-6082$

(3) Sumida, K.; Rogow, D. L; Mason, J. A.; McDonald, T. M.; Bloch, E. D.; Herm, Z. R.; Bae, T.-H.; Long, J. R. Carbon Dioxide Capture in Metal-Organic Frameworks. Chem. Rev. 2012, 112, 724-781.

(4) Lin, L.-C.; Berger, A. H.; Martin, R. L.; Kim, J.; Swisher, J. A.; Jariwala, K.; Rycroft, C. H.; Bhown, A. S.; Deem, M. W.; Haranczyk, M.; Smit, B. In Silico Screening of Carbon-Capture Materials. Nat. Mater. 2012, 11, 633-641.

(5) Smit, B.; Reimer, J. R.; Oldenburg, C. M.; Bourg, I. C. Introduction to Carbon Capture and Sequestration; Imperial College Press: London, 2014; Chapter 1, pp 1-19.

(6) Rappe, A.; Casewit, C. UFF, a Full Periodic Table Force Field for Molecular Mechanics and Molecular Dynamics Simulations. J. Am. Chem. Soc. 1992, 2, 10024-10035.

(7) Mayo, S.; Olafson, B.; Goddard, W. DREIDING: A Generic Force Field for Molecular Simulations. J. Phys. Chem. 1990, 94, 88978909.

(8) Dincă, M.; Long, J. R. Hydrogen Storage in Microporous MetalOrganic Frameworks with Exposed Metal Sites. Angew. Chem., Int. Ed. 2008, 47, 6766-6779.

(9) Caskey, S. R.; Wong-Foy, A. G.; Matzger, A. J. Dramatic Tuning of Carbon Dioxide Uptake via Metal Substitution in a Coordination Polymer with Cylindrical Pores. J. Am. Chem. Soc. 2008, 130, 1087010871.

(10) Britt, D.; Furukawa, H.; Wang, B.; Glover, T. G.; Yaghi, O. M. Highly Efficient Separation of Carbon Dioxide by a Metal-Organic 
Framework Replete with Open Metal Sites. Proc. Natl. Acad. Sci. U.S.A. 2009, 106, 20637-20640.

(11) Dietzel, P. D. C.; Besikiotis, V.; Blom, R. Application of MetalOrganic Frameworks with Coordinatively Unsaturated Metal Sites in Storage and Separation of Methane and Carbon Dioxide. J. Mater. Chem. 2009, 19, 7362-7370.

(12) Krishna, R.; Van Baten, J. M. In Silico Screening of MetalOrganic Frameworks in Separation Applications. Phys. Chem. Chem. Phys. 2011, 13, 10593-10616.

(13) Chen, L.; Morrison, C. A.; Düren, T. Improving Predictions of Gas Adsorption in Metal-Organic Frameworks with Coordinatively Unsaturated Metal Sites: Model Potentials, Ab Initio Parameterization, and GCMC Simulations. J. Phys. Chem. C 2012, 116, 18899-18909.

(14) Fang, H.; Kamakoti, P.; Zang, J.; Cundy, S.; Paur, C.; Ravikovitch, P. I.; Sholl, D. S. Prediction of $\mathrm{CO}_{2}$ Adsorption Properties in Zeolites Using Force Fields Derived from Periodic Dispersion-Corrected DFT Calculations. J. Phys. Chem. C 2012, 116, 10692-10701.

(15) Zang, J.; Nair, S.; Sholl, D. S. Prediction of Water Adsorption in Copper-Based Metal-Organic Frameworks Using Force Fields Derived from Dispersion-Corrected DFT Calculations. J. Phys. Chem. C 2013, 117, 7519-7525.

(16) Dzubak, A. L.; Lin, L.-C.; Kim, J.; Swisher, J. A.; Poloni, P.; Maximoff, S. N.; Smit, B.; Gagliardi, L. Ab Initio Carbon Capture in Open-Site Metal-Organic Frameworks. Nat. Chem. 2012, 4, 810-816.

(17) Hagberg, D.; Karlstrom, G.; Roos, B. O.; Gagliardi, L. The Coordination of Uranyl in Water: A Combined Quantum Chemical and Molecular Simulation Study. J. Am. Chem. Soc. 2005, 127, 1425014256.

(18) Engkvist, O.; Astrand, P. O.; Karlstrom, G. Accurate Intermolecular Potentials Obtained from Molecular Wave Functions: Bridging the Gap between Quantum Chemistry and Molecular Simulations. Chem. Rev. 2000, 100, 4087-4108.

(19) Queen, W.; Brown, C.; Britt, D. Site-Specific $\mathrm{CO}_{2}$ Adsorption and Zero Thermal Expansion in an Anisotropic Pore Network. J. Phys. Chem. C 2011, 115, 24915-24919.

(20) Liu, J.; Benin, A. I.; Furtado, A. M. B.; Jakubczak, P.; Willis, R. R.; LeVan, M. D. Stability Effects on $\mathrm{CO}_{2}$ Adsorption for the DOBDC Series of Metal-Organic Frameworks. Langmuir 2011, 27, 1145111456.

(21) Yu, J.; Balbuena, P. Water Effects on Postcombustion $\mathrm{CO}_{2}$ Capture in Mg-MOF-74. J. Phys. Chem. C 2013, 117, 3383-3388.

(22) Lee, K.; Murray, É. D.; Kong, L.; Lundqvist, B. I.; Langreth, D. C. Higher-Accuracy Van Der Waals Density Functional. Phys. Rev. $B$ 2010, 82, 081101.

(23) Giannozzi, P.; Baroni, S.; Bonini, N.; Calandra, M.; Car, R.; Cavazzoni, C.; Ceresoli, D.; Chiarotti, G. L.; Cococcioni, M.; Dabo, I.; Dal Corso, A.; de Gironcoli, S.; Fabris, S.; Fratesi, G.; Gebauer, R.; Gerstmann, U.; Gougoussis, C.; Kokalj, A.; Lazzeri, M.; Martin-Samos, L.; Marzari, N.; Mauri, F.; Mazzarello, R.; Paolini, S.; Pasquarello, A.; Paulatto, L.; Sbraccia, C.; Scandolo, S.; Sclauzero, G.; Seitsonen, A. P.; Smogunov, A.; Umari, P.; Wentzcovitch, R. M. QUANTUM ESPRESSO: A Modular and Open-Source Software Project for Quantum Simulations of Materials. J. Phys.: Condens. Matter 2009, 21, 395502.

(24) Frenkel, D.; Smit, B. Understanding Molecular Simulations: from Algorithms to Applications, 2nd ed.; Academic Press: San Diego, CA, 2002; Chapter 12, pp 291-300.

(25) Campañá, C.; Mussard, B.; Woo, T. Electrostatic potential derived atomic charges for periodic systems using a modified error functional. J. Chem. Theory Comput. 2009, 5, 2866-2878.

(26) Potoff, J. J.; Siepmann, J. I. Vapor-Liquid Equilibria of Mixtures Containing Alkanes, Carbon Dioxide, and Nitrogen. AIChE J. 2001, 47, 1676-1682.

(27) Harris, J.; Yung, K. Carbon Dioxide's Liquid-Vapor Coexistence Curve and Critical Properties as Predicted by a Simple Molecular Model. J. Phys. Chem. 1995, 99, 12021-12024.

(28) Mason, J. A.; Sumida, K.; Herm, Z. R.; Krishna, R.; Long, J. R. Evaluating Metal-Organic Frameworks for Post-combustion Carbon
Dioxide Capture via Temperature Swing Adsorption. Energy Environ. Sci. 2011, 4, 3030-3040.

(29) Grimme, S. Semiempirical GGA-type Density Functional Constructed with a Long-range Dispersion Correction. J. Comput. Chem. 2006, 27, 1787-1799.

(30) Lin, L.-C.; Kim, J.; Kong, X.; Scott, E.; McDonald, T. M.; Long, J. R.; Reimer, J. A.; Smit, B. Understanding $\mathrm{CO}_{2}$ Dynamics in MetalOrganic Frameworks with Open Metal Sites. Angew. Chem., Int. Ed. 2013, 52, 4410-4413.

(31) Kong, X.; Scott, E.; Ding, W.; Mason, J. A; Long, J. R.; Reimer, J. A. $\mathrm{CO}_{2}$ Dynamics in a Metal-Organic Framework with Open Metal Sites. J. Am. Chem. Soc. 2012, 134, 14341-14344.

(32) Canepa, P.; Nijem, N.; Chabal, Y. J.; Thonhauser, T. Diffusion of Small Molecules in Metal Organic Framework Materials. Phys. Rev. Lett. 2013, 110, 026102.

(33) Yu, D.; Yazaydin, a. O.; Lane, J. R.; Dietzel, P. D. C.; Snurr, R. Q. A Combined Experimental and Quantum Chemical Study of $\mathrm{CO}_{2}$ Adsorption in the Metal-Organic Framework CPO-27 with Different Metals. Chem. Sci. 2013, 4, 3544-3556.

(34) Horn, H. W.; Swope, W. C.; Pitera, J. W.; Madura, J. D.; Dick, T. J.; Hura, G. L.; Head-Gordon, T. Development of an Improved Four-Site Water Model for Biomolecular Simulations: TIP4P-Ew. J. Chem. Phys. 2004, 120, 9665-9678.

(35) Yang, D.-A.; Cho, H.-Y.; Kim, J.; Yang, S.-T.; Ahn, W.-S. $\mathrm{CO}_{2}$ Capture and Conversion Using Mg-MOF-74 Prepared by a Sonochemical Method. Energy Environ. Sci. 2012, 5, 6465-6473.

(36) Schoenecker, P. M.; Carson, C. G.; Jasuja, H.; Flemming, C. J. J.; Walton, K. S. Effect of Water Adsorption on Retention of Structure and Surface Area of Metal-Organic Frameworks. Ind. Eng. Chem. Res. 2012, 51, 6513-6519.

(37) Glover, T. G.; Peterson, G. W.; Schindler, B. J.; Britt, D.; Yaghi, O. MOF-74 Building Unit has a Direct Impact on Toxic Gas Adsorption. Chem. Eng. Sci. 2011, 66, 163-170.

(38) Canepa, P.; Arter, C.; Conwill, E. High-throughput Screening of Small-Molecule Adsorption in MOF. J. Mater. Chem. A 2013, 1, $13597-13604$.

(39) Valenzano, L.; Civalleri, B.; Chavan, S.; Palomino, G. T.; Area, C. O.; Bordiga, S. Computational and Experimental Studies on the Adsorption of $\mathrm{CO}, \mathrm{N}_{2}$, and $\mathrm{CO}_{2}$ on Mg-MOF-74. J. Phys. Chem. C 2010, 114, 11185-11191. 